\title{
La electrólisis percutánea y su eficacia en el tratamiento de tendinopatías en la extremidad inferior. Revisión sistemática
}

\section{Percutaneous electrolysis and its efficacy in the treatment of tendinopathies in the lower limb. Systematic review}

Andrea Elizabeth Villamarín Arévalo. ${ }^{1}$, Juan Carlos Muyulema Allaica. ${ }^{2}$, Robert Paul Culqui García. ${ }^{3}$ \& Marlon Esteban Sandoval Tulcán. ${ }^{4}$

Recibido: 16-08-2019 / Revisado: 17-09-209 /Aceptado: 28-09-2019/ Publicado: 04-10-2019

\begin{abstract}
.
DOI: https://doi.org/10.33262/cienciadigital.v3i4.966

Nowadays, the tendinopathies world conception has tolerated big changes. The separation of the theories that declare the tendinopathies how inflammatory disorders, theories that allude a pathophysiology of continuing character based on degenerative changes, led to the emergence of physical therapy techniques such as percutaneous invasive electrolysis, which seek to break with this process of degeneration, allowing the recovery of the tissue. The overall objective of this systematic review is to study the efficacy and scientific evidence of the technique of percutaneous electrolysis applied to the treatment of tendinopathies in the lower lamb, to compare the obtained results. For which, it was made a systematic search of studies in 6 databases, using the key words from: patellar tendinopathy with electrolysis, the treatment, physical therapy and rehabilitation, which offered 28 articles, of which, 8 were used in the review, specifically, studies of 5 case series and 2 prospective studies in rats and 1 clinical case. Percutaneous electrolysis, showed a functional improvement and in the

1 Universidad Estatal de Bolívar, Facultad de Ciencias de Salud y del Ser Humano. Guaranda, Ecuador,

${ }^{2}$ Pontificia Universidad Católica del Ecuador Sede Ambato (PUCESA), Escuela de Diseño Industrial.

Ambato, Ecuador., juanca327@ hotmail.com

${ }^{3}$ Universidad Estatal de Bolívar, Facultad Ciencias Administrativas Gestión Empresarial e Informática. Guaranda, Ecuador, rculqui@ueb.edu.ec

${ }^{4}$ Hospital Metropolitano, Terapia Física. Quito, Ecuador)., marlonsandovalt@hotmail.com
\end{abstract} aeva85@hotmail.com 
painful feeling, to be combined with eccentric exercise therapies. The therapeutic treatment of tendinopathies in the lower limb through percutaneous electrolysis, provides very promising results, however, to develop the consequence of these results, require the input of a substantially larger amount of studies with a more sophisticated methodology, which support your scientific evidence.

Keywords: Percutaneous electrolysis, efficacy, lower limb, systematic review, tendinopathies.

\section{Resumen.}

En los últimos años, la concepción que se tenía de las tendinopatías ha tolerado grandes cambios. La separación de las teorías que declaran a las tendinopatías como trastornos inflamatorios, a teorías que aluden una fisiopatología de carácter continúo basado en cambios degenerativos, propició la aparición de técnicas de fisioterapia invasiva como la electrólisis percutánea, que buscan romper con este proceso de degeneración, permitiendo la recuperación del tejido. El objetivo general de esta revisión sistemática es, estudiar la eficacia y evidencia científica de la técnica del electrólisis percutánea aplicada al tratamiento de tendinopatías en la extremidad inferior, para comparar los resultados obtenidos. Para lo cual, se realizó una búsqueda sistemática de estudios en 6 bases de datos, empleando las palabras clave: patellar tendinopathy unido con electrolysis, treatment, physical therapy y rehabilitation, lo cual ofreció 28 artículos, de los cuales, 8 se emplearon en la revisión, concretamente, 5 estudios de series de casos y 2 estudios prospectivos en ratas y 1 caso clínico. La electrólisis percutánea, mostró una mejora funcional y en la sensación dolorosa, al ser combinada con terapias de ejercicios excéntricos. El tratamiento terapéutico de las tendinopatías en la extremidad inferior mediante electrólisis percutánea, brinda resultados muy promisorios, sin embargo, para establecer la consecuencia de estos resultados, requiere del aporte de una cantidad sustancialmente mayor de estudios con una metodología más sofisticada, que sustenten su evidencia científica.

Palabras claves: Electrólisis percutánea, eficacia, extremidad inferior, revisión sistemática, tendinopatías.

\section{Introducción.}

El dolor localizado en el tendón, característicamente en el tendón de Aquiles y el tendón rotuliano, distinguido como tendinopatía es estrechamente común en individuos practicantes de algún tipo de actividad física, ya sea a nivel más o menos profesional como en aquellas que lo hacen de forma recreacional (Singh, Calafi, Diefenbach, Kreulen, \& Giza, 2017; Sánchez \& Seoane, 2018). Sin embargo, y según diversos estudios sobre la temática, se ha demostrado que personas físicamente inactivas también lo sufren (Weber \& Buchhorn, 2017; Lim \& Wong, 2018; Splittgerber \& Ihm, 2019). Por lo tanto, se puede aseverar que, la actividad física no puede ser asociada directamente 
a la histopatología y que el ejercicio físico no infiere en la provocación de los síntomas o ser el causante de la lesión (Lui, 2017; Yang, Lu, \& Qu, 2018).

Los avances acreditados en la comprensión del proceso de la enfermedad razonan que el sobreuso induce esta condición, pero la etiología y la patogenia no están científicamente depuradas, sin llegar a un consenso entre los investigadores (Jennings, Liew, \& Marine, 2019). La mayoría de las tendinopatías constan asociadas a un defecto en el metabolismo o a la estructura de la matriz celular de los tenacitos, colocando en riesgo su elasticidad y resistencia (Ackermann \& Hart, 2016; Yang, Lu, \& Qu, 2018).

Es probable que, también estén relacionados los factores intrínsecos como los errores de entrenamiento (maquinas, técnicas o progresiones inadecuadas), la temperatura (el frio hace el tendón más rígido) o la superficie sobre la que se corre (Cook \& Purdam, 2009; Scott, Backman, \& Speed, 2015). Conjuntamente, de los factores extrínsecos, también existen factores intrínsecos, que facilitan el progreso de las tendinopatías, como la genética, la edad, el sexo, la circulación, y la biomecánica y composición del tendón, pueden someter a estas estructuras a cargas excesivas (Scott, Backman, \& Speed, 2015; Ackermann \& Hart, 2016). Tres son las hipótesis que se asocian a la rotura de un tendón: la teoría mecánica, la vascular, y la neural.

Actualmente, autores como Arnal-Burró et al., (2015); Ackermann \& Hart (2016); De Carbo \& Bullock (2017); Sánchez \& Seoane (2018); Jennings, Liew, \& Marine (2019), que tras los hallazgos histopatológicos y ecográficos estudiados están llegando a la conclusión de que la mayoría de las tendinopatías están asociadas y relacionadas a un único factor, que es la existencia de un proceso degenerativo previo a la rotura.

La tendinopatía degenerativa está ampliamente descrita en la literatura como una progresión de la desorganización de la matriz de colágeno, cambios en las células y aparición de neovascularización (Cook et al., 2017). Aparecen áreas de muerte celular por apoptosis, trauma o fallo de los tenocitos (Ackermann \& Hart, 2016; Yang, Lu, \& Qu, 2018). La capacidad de reversibilidad de los cambios patológicos es muy remota (De Almeida, Da Silva, \& Ronzio, 2019).

El proceso de regeneración tendinosa, es lo que conocemos como la sustitución del tejido afecto por otro con las mismas características al primitivo (Freed, Ellis, Johnson, \& Haddon, 2019). Una vez que estimulamos el tejido dañado mediante la electrolisis percutánea, se provoca una respuesta inflamatoria, cuya función es producir la regeneración tisular mediante tres fases apropiadamente definidas en la literatura: fase inflamatoria, fase de reparación fibroblástica y fase de remodelación/maduración (Carvajal, Álvarez, Medina, \& Minaya, 2016; Sánchez \& Seoane, 2018).

En los últimos años, la aplicación de corriente galvánica por medio de una aguja de acupuntura se ha empleado en el tratamiento de las tendinopatías (Sánchez \& Seoane, 2018). Está técnica es nombrada con diversos nombres comerciales: Electrólisis Percutánea Intratisular (EPI ${ }^{\circledR}$ ), 
Electrólisis Percutánea Terapéutica $\left(\mathrm{EPTE}^{\circledR}\right)$ o Electrolisis Percutánea Musculoesquética (EPM); siendo el término común electrólisis percutánea (Arias-Buria, y otros, 2015). Todas estas técnicas se fundamentan en los mismos principios, difiriendo unas de otras esencialmente en la intensidad de la corriente y el tiempo durante el cual esta es aplicada (Carvajal, Álvarez, Medina, \& Minaya, 2016). Por un lado, la técnica EPI ${ }^{\circledR}$ utiliza intensidades mayores (3-6 mA) durante un corto periodo de tiempo (alrededor de 4 segundos), mientras que la técnica $\mathrm{EPTE}^{\circledR}$, se distingue por intensidades menores $(350 \mu \mathrm{A})$ durante periodos de tiempo más largos (1.2 minutos) (Arias-Buria et al., 2015). A pesar de que la EPTE ${ }^{\circledR}$ presume de provocar menos molestia en el paciente, no se han perpetrado suficientes estudios sobre su eficacia, a diferencia de la EPI ${ }^{\circledR}$ (Valera-Garrido, Minaya-Muñoz, \& Medina-Mirapeix, 2014; Mattiussi \& Moreno, 2016; Rodríguez \& Mayordomo, 2017).

La electrolisis percutánea consiste en la trasferencia de una corriente galvánica a través de una aguja de acupuntura de 0,30 a 0,33mm de diámetro, la cual penetrará la piel del paciente hasta llegar al tejido diana, induciendo a una reacción electroquímica, que se traducirá en la destrucción del tejido lesionado y en un proceso inflamatorio local (Mattiussi \& Moreno, 2016).

Para aplicar la electrolisis percutánea, se manipula un aparato generador de corriente galvánica concreto. Este aparato consta de dos electrodos de caracteres distintos. El electrodo activo es el negativo, cuyo terminal consta de una aguja de acupuntura, a través de la cual se realizará la punción. El electrodo positivo, por otra parte, es un electrodo manual con forma cilíndrica, que el paciente debe sujetar con su mano para, de esta manera, cerrar el circuito eléctrico (Moreno, Mattiussi, \& Núñez, 2016).

$\mathrm{Al}$ actuar esta corriente hace que el cloruro de sodio $(\mathrm{NaCl})$ y el agua $\left(\mathrm{H}_{2} \mathrm{O}\right)$, se descompongan en sus elementos químicos constitutivos, los cuales se agrupan entre ellos para formar sustancias nuevas. Las nuevas sustancias que se forman a partir de la sal y el agua son hidróxido de sodio $(\mathrm{NaOH})$, gas hidrógeno $\left(\mathrm{H}_{2}\right)$ y gas cloro $\left(\mathrm{Cl}_{2}\right)$. El $\mathrm{NaOH}$, es lo que conocemos comúnmente como hidróxido sódico o hidrato de sodio, también conocido como soda cáustica (en casi toda Latinoamérica) o sosa cáustica (en México y España), es un hidróxido cáustico que va a actuar sobre el tendón provocando la destrucción completa de las células dañadas por la degradación del colágeno y la sustancia mixoide (García, De La Cruz, Naranjo, \& Albornoz, 2018).

La electrolisis es un proceso químico en el que no existe ni cocción ni electrocución del tejido. Al aplicarla sobre la región lesionada, se va a producir una destrucción del tejido que provoca una respuesta inflamatoria para su reparación.

Por lo tanto, según este principio, la electrólisis percutánea, mediante dosis terapéuticas de corriente continua aplicadas en tejidos blandos, busca producir una permutación en los valores de pH en la interfase electrodo-tejido de la zona tratada, lo que destruirá el tejido sin que las células sanas se vean afectadas (De Almeida, Da Silva, \& Ronzio, 2019). Este tejido destruido es metabolizado por el propio organismo a través de la fagocitosis inducida (Sánchez \& Seoane, 
2018). Además, la acción catódica de la corriente continua, admitirá la atracción de los tenoblastos a la zona, excitando su proliferación y la síntesis de nuevo colágeno.

Los efectos inmediatos sobre el tejido a continuación de la aplicación del electrolisis dependerán del electrodo activo. Al utilizar como electrodo activo el cátodo (negativo), dará lugar a una irritación y destrucción del tejido. Esto causará una modificación del pH del entorno, favoreciendo la invasión capilar que aportará oxígeno y nutrientes, normalizando el $\mathrm{PO}_{2}$ (De la Cruz, Albornoz, García, \& Naranjo, 2016; Rodríguez \& Mayordomo, 2017). El pico máximo de inflamación en los tendones corresponde al quinto día después de la intervención y puede durar hasta quince días (Sánchez \& Seoane, 2018; Jennings, Liew, \& Marine, 2019).

El objetivo general de esta revisión sistemática es, estudiar la eficacia y evidencia científica de la técnica del electrólisis percutánea aplicada al tratamiento de tendinopatías en la extremidad inferior, para comparar los resultados obtenidos. La revisión se realizó conforme a lo recogido en el protocolo internacional para revisiones sistemáticas y metaanálisis PRISMA, definiendo el acrónimo PICO cuyas siglas responden a los términos que deben ser incluidos en dicha cuestión: Population (Población/Participantes), Intervention (Intervención), Comparison (Comparación) y Outcome (Resultado), que define los puntos críticos sobre los que se centra dicha revisión (Urrútia \& Bonfill, 2010; Sánchez, Esquirol, \& Dalmau, 2016).

\section{Metodología.}

Se realizó una búsqueda en las bases de datos entre las que se incluyeron: Scopus, Web of Science, PubMed, SciELO, Dialnet y PEDro. Los términos utilizados como las palabras clave fueron: patellar tendinopathy unido con electrolysis, treatment, physical therapy y rehabilitation. Se seleccionaron artículos en idioma inglés o español, con antigüedad máxima de 5 años.

Se incluyeron todos los artículos que cumplían los siguientes criterios de inserción:

- Artículos que empleaban la técnica Electrólisis Percutánea Terapéutica (EPTE) y/o Electrolisis Percutánea Intratisular (EPI) en distintos tipos de lesiones tendinosas, y que afectaban a la extremidad inferior.

- Artículos que investigaban en cualquier especie (animal o humana) con tendinopatía en la extremidad inferior, con aplicación del electrolisis percutánea, y que puntualicen la presencia o no de curación, el tiempo y el número de aplicaciones.

- Artículos que aportaban datos clínicos, realizados al menos con un paciente o una sola lesión.

Para analizar la calidad metodológica de cada artículo, se utilizaron aspectos actuales y novedosos acordes a lo recogido en el protocolo internacional para revisiones sistemáticas y meta análisis 
PRISMA (Preferred Reporting Items for Systematic reviews and Meta-Analyses), definiendo el acrónimo PICO cuyas siglas responden a los términos que deben ser incluidos en dicha cuestión: Population (Población/Participantes), Intervention (Intervención), Comparison (Comparación) y Outcome (Resultado), que define los puntos críticos sobre los que se centra dicha revisión (Urrútia \& Bonfill, 2010; Sánchez, Esquirol, \& Dalmau, 2016).

El estudio partió desde el inicio mismo del proceso (los registros o las citas identificados en las búsquedas realizadas en cada una de las diferentes 6 bases de datos utilizadas), continúa por el número total de registros o citas y termina con los estudios individuales incluidos en la síntesis cualitativa (revisión sistemática) y cuantitativa (metaanálisis).

Los cuatro autores del manuscrito valoraron por separado todos los artículos académicos e investigativos: previos a su inclusión y no se tomaron en cuenta los artículos de opinión o divulgación que sumaron un total de 12. Conjuntamente, fueron encontrados 3 artículos que no se refirieron a las tendinopatías en la extremidad inferior, más bien trataron las epicondilitis y lesiones de los músculos pectorales, no obstante, estuvieron asistidas con la técnica de EPTE y/o EPI. Otros 5 artículos fueron del mismo modo descartados puesto que, si bien empleaban la técnica de electrolisis en la extremidad inferior, lo utilizaron sobre patologías no tendinosas. Por tanto, de los 28 artículos detectados acordes con los criterios de inclusión, solo 8 artículos trataban el objetivo fundamental de este trabajo sobre estudiar la eficacia y evidencia científica de la técnica del electrólisis percutánea aplicada al tratamiento de tendinopatías en la extremidad inferior.

Finalmente, se realizó un análisis descriptivo de los datos obtenidos en los estudios valorados, analizados e incluidos en el estudio, en los que se registró el tipo de estudio realizado, la patología y el número de pacientes tratados, el número de sesiones realizadas, la presencia o no de ejercicios excéntricos y los resultados obtenidos; así como se discutieron las limitaciones, la eficacia de la electrolisis percutánea y futuras líneas de investigación que puedan resolver las lagunas presentes en la actualidad.

\section{Resultados.}

La Figura 1 muestra el flujo de artículos que se valoraron y analizaron durante la realización de la presente revisión sistemática. De los 8 artículos finalmente utilizados, 1 era caso clínico (Sánchez \& Seoane, 2018), 5 eran estudios de series de casos (Yeo, Kendall, \& Jayaraman, 2016; Abat, Gelber, Polidori, Monllau, \& Sanchez-Ibaez, 2015; Abat, y otros, 2016; Carvajal, Álvarez, Medina, \& Minaya, 2016; Lang, y otros, 2017), y dos trataban tendinopatías con electrolisis en los miembros inferiores de las ratas que se utilizaron para el estudio (De Souza, y otros, 2015; Rodríguez \& Mayordomo, 2017). 


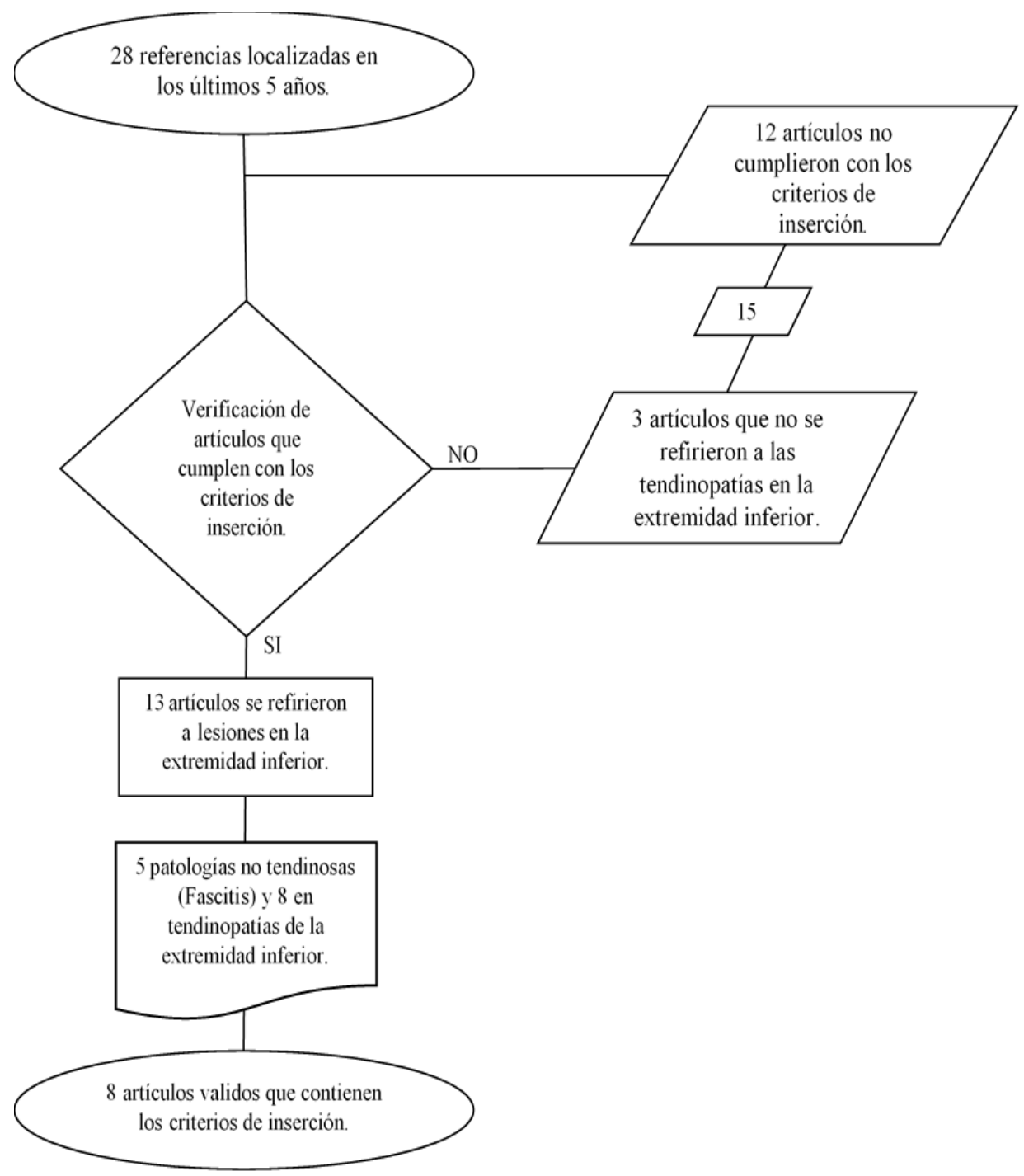

Figura 1. Flujo de artículos valorados y analizados en la revisión sistemática.

Fuente: Elaboración propia

Los ocho artículos seleccionados tratan afecciones tendinosas de la extremidad inferior con electrolisis (4 de ellos trataban tendinopatías del tendón de Aquiles y los otros 4 se relacionaban con patologías del tendón rotuliano) (Tabla 1). Todos refieren mejoría tras al menos 4 semanas de tratamiento y hasta 12 semanas, con la aplicación de 1 a 6 sesiones por semana, aunque dos trabajos experimentales en ratas refieren mejoría tras 2 y 4semanas de la intervención, con la aplicación de 14 y 6 sesiones por semana respectivamente (Rodríguez \& Mayordomo, 2017; De Souza, y otros, 2015). 
Tabla 1. Revisión descriptiva de la eficacia del electrolisis percutánea (relación beneficio-tiempo)

\begin{tabular}{|c|c|c|c|c|c|c|}
\hline Autores & $\begin{array}{c}\text { Patología } \\
\text { tratada }\end{array}$ & $\begin{array}{l}\text { Número de } \\
\text { Pacientes }\end{array}$ & $\begin{array}{c}\text { ejercicios } \\
\text { excéntricos }\end{array}$ & $\begin{array}{l}\text { Numero de } \\
\text { sesiones }\end{array}$ & $\begin{array}{c}\text { Tiempo } \\
\text { promedio de } \\
\text { curación }\end{array}$ & Eficacia \\
\hline $\begin{array}{l}\text { (De Souza, y otros, } \\
\text { 2015) }\end{array}$ & $\begin{array}{c}\text { Tendinopatía } \\
\text { de Aquiles }\end{array}$ & $\begin{array}{l}15 \text { (estudio } \\
\text { experimental } \\
\text { en ratas) }\end{array}$ & $\mathrm{Si}$ & $\begin{array}{l}6 \text { sesiones } \\
\text { semanales }\end{array}$ & 4 semanas & $\mathrm{Si}$ \\
\hline $\begin{array}{l}\text { (Yeo, Kendall, \& } \\
\text { Jayaraman, 2016) }\end{array}$ & $\begin{array}{c}\text { Tendinopatía } \\
\text { de Aquiles }\end{array}$ & 21 & $\mathrm{Si}$ & $\begin{array}{l}6 \text { sesiones } \\
\text { semanales }\end{array}$ & 4 semanas & $\mathrm{Si}$ \\
\hline $\begin{array}{l}\text { (Rodríguez \& } \\
\text { Mayordomo, 2017) }\end{array}$ & $\begin{array}{c}\text { Tendinopatía } \\
\text { de Aquiles }\end{array}$ & $\begin{array}{l}20 \text { (estudio } \\
\text { experimental } \\
\text { en ratas) }\end{array}$ & No & 14 sesiones & 2 semanas & $\mathrm{Si}$ \\
\hline $\begin{array}{l}\text { (Sánchez \& } \\
\text { Seoane, 2018) }\end{array}$ & $\begin{array}{c}\text { Tendinopatía } \\
\text { de Aquiles }\end{array}$ & 1 & $\mathrm{Si}$ & $\begin{array}{l}4 \text { sesiones } \\
\text { semanales }\end{array}$ & 10 semanas & $\mathrm{Si}$ \\
\hline $\begin{array}{l}\text { (Abat, Gelber, } \\
\text { Polidori, Monllau, } \\
\text { \& Sanchez-Ibaez, } \\
\text { 2015) }\end{array}$ & $\begin{array}{c}\text { Tendinopatía } \\
\text { rotuliana }\end{array}$ & 40 & $\mathrm{Si}$ & $\begin{array}{l}4 \text { sesiones } \\
\text { semanales }\end{array}$ & 12 semanas & $\mathrm{Si}$ \\
\hline $\begin{array}{l}\text { (Abat, y otros, } \\
\text { 2016) }\end{array}$ & $\begin{array}{c}\text { Tendinopatía } \\
\text { rotuliana }\end{array}$ & 30 & $\mathrm{Si}$ & $\begin{array}{l}3 \text { sesiones } \\
\text { semanales }\end{array}$ & 8 semanas & $\mathrm{Si}$ \\
\hline $\begin{array}{l}\text { (Carvajal, Álvarez, } \\
\text { Medina, \& } \\
\text { Minaya, 2016) }\end{array}$ & $\begin{array}{c}\text { Tendinopatía } \\
\text { rotuliana }\end{array}$ & 19 & No & $\begin{array}{l}6 \text { sesiones } \\
\text { semanales }\end{array}$ & 6 semanas & $\mathrm{Si}$ \\
\hline (Lang, et al., 2017) & $\begin{array}{c}\text { Tendinopatía } \\
\text { rotuliana }\end{array}$ & 30 & $\mathrm{Si}$ & $\begin{array}{l}1 \text { sesión } \\
\text { semanal }\end{array}$ & 12 semanas & $\mathrm{Si}$ \\
\hline
\end{tabular}

\section{Fuente: Elaborado propia}

Si analizamos los datos por pacientes tratados y no por trabajos podemos contabilizar un total de 141 pacientes sometidos a esta terapia novedosa, casi todos procedentes del entorno deportivo, de los cuales todos han conseguido una recuperación completa y funcional en un tiempo menor con respecto a otros tratamientos convencionales (cuyo tiempo medio de recuperación supera los tres meses y la eficacia no supera el 60\%) (Mani-Babu, Morrissey, Waugh, Screen, \& Barton, 2015; Shakked \& Raikin, 2017; Korakakis, Whiteley, Tzavara, \& Malliaropoulos, 2018), frente al promedio de curación con la electrolisis que tiene límites de 4 a 12 semanas y permitiendo al paciente retomar su actividad diaria y/o deportiva en tiempo menor. Del mismo modo los trabajos que realizan un experimento en ratas con el tendón de Aquiles roto, muestra una mejoría significativa al cabo de 2 y 4 semanas de tratamiento (Rodríguez \& Mayordomo, 2017; De Souza, y otros, 2015).

El 75\% de los autores considerados en este estudio optan, para la aplicación de esta terapia, por una combinación con ejercicios excéntricos. En el trabajo de Carvajal, Álvarez, Medina, \& Minaya (2016) realizado en tendinopatía rotuliana optan por una terapia única con electrolisis alcanzando mejoría con un tiempo de recuperación completa que está dentro de los parámetros descritos para 
todos los artículos revisados (de 4 a 12 semanas) (tabla 1). En cualquier caso, este tiempo resulta menor que los tres meses mínimos que se necesitan aplicando otras técnicas convencionales (tratamientos farmacológicos y biológicos, tratamientos rehabilitadores, técnicas de frío/calor, ondas de choque extracorpóreas, entre otras) (Zwiers, Wiegerinck, \& Van Dijk, 2016; Ögüt \& Yontar, 2017).

Del total de los 141 pacientes (sumados cada uno de los pacientes tratados en cada uno de los estudios revisados) sometidos a electrolisis, un total de 122 pacientes han combinado las terapias electrolíticas con ejercicios excéntricos. El número de sesiones por semana aplicadas cuando se combinan los tratamientos es menor de 6, mientras que cuando se aplica la electrolisis solo, el número de sesiones requeridas supera las 6 semanas y no mejora el tiempo de recuperación (Tabla 1). Estos datos evidencian que la técnica combinada de electrolisis y ejercicios terapéutico de estiramiento están substancialmente indicados en el tratamiento de lesiones, puesto que está confirmado que el músculo se activa más durante el estiramiento excéntrico, y por ello provoca un proceso de reparación y regeneración en las lesiones músculo-tendinosas, bien sean contracturas, distensiones, roturas de fibras o tendinosis. Bajo este criterio, terapias electrolíticas combinadas con ejercicios excéntricos, parece ser, el método más efectivo puesto que permite la alineación de las nuevas fibras de colágeno neo formadas mediante la elongación del tendón.

Sin embargo, hay que resaltar en este punto que en los trabajos revisados se muestra que el uso de un ecógrafo parece ser muy útil para asegurar una aplicación correcta (Abat, Gelber, Polidori, Monllau, \& Sanchez-Ibaez, 2015; Lang, y otros, 2017). Por tanto, el tratamiento de la tendinopatía rotuliana y la tendinopatía del tendón de Aquiles con electrolisis percutánea podría ser efectivo a corto plazo, no obstante, algunos autores refieren que su aplicación es dolorosa (Yeo, Kendall, \& Jayaraman, 2016; Abat, y otros, 2016; Sánchez \& Seoane, 2018).

\section{Discusión.}

\section{Limitaciones de la revisión}

A la hora de desarrollar y ampliar la revisión, nos tropezamos con una serie de limitaciones, que imposibilitaron la realización de una investigación más exhaustiva de la materia.

Una de las limitaciones, fue que se restringió la búsqueda a artículos académicos e investigativos de acceso gratuito, por lo que aquellos que demandaban de un pago para ser leídos fueron eliminados, reduciendo así el número de artículos empleados en la revisión.

Las palabras clave empleadas para la búsqueda de artículos, a pesar de ser bastante genéricas, fueron de un número limitado en función de cumplir criterios de inserción. Además, la búsqueda se realizó en 6 bases de datos, que, a pesar de ser un número moderado de bases de datos, estas no aglomeran la totalidad de estudios perpetrados en la materia. Por lo que, la ampliación de las palabras clave y de las bases de datos, podrían brindar algunos estudios más. 


\section{Limitaciones de los estudios}

Las principales limitaciones encontradas en los artículos académicos e investigativos son:

1. El tamaño de la muestra. El tamaño de la muestra en los estudios recogidos rondó entre 1 y los 40 sujetos. El reducido tamaño muestral, puede conllevar a una baja significación estadística de los resultados, provocando un bajo nivel de evidencia de los artículos.

2. Diseño de estudio. Al igual que el tamaño muestral, el diseño del estudio puede reducir de modo considerable el nivel de evidencia del estudio. Los estudios examinados en esta revisión sistemática, fueron en su mayoría una serie de casos (5 estudios), estudios prospectivos en ratas ( 2 estudios) y ensayos clínicos aleatorizados (1 estudio).

3. La falta de un grupo control. Se constató solamente 3 estudios que manejaron un grupo de control. En uno de los estudios, el grupo control no recibía tratamiento de ningún tipo, comprobando así que los resultados no se debían simplemente al paso del tiempo. Los otros dos estudios, manejaban un grupo control que era tratado mediante un programa de tratamiento base, como ejercicios excéntricos y estiramientos, concurriendo el grupo de estudio tratado por este mismo tratamiento, más electrólisis percutánea. Esta metodología, permitió determinar si la electrolisis percutánea generaba una mejora más sustancial, probando así su eficiencia.

4. La falta de cotejo con un grupo placebo. Ninguno de los estudios que establecieron la revisión sistemática, ofreció un grupo tratado mediante un placebo, lo cual no nos consiente establecer si las mejoras de resultados del electrolisis percutánea se deben a una mejora de la patología, o a un efecto placebo originada por esta.

5. El hecho de que no se estudie la electrolisis percutánea de forma aislada, sino combinada con terceras terapias. De los estudios recogidos en la revisión, exclusivamente dos evaluaron la eficacia del electrolisis percutánea como tratamiento único. Esto puede provocar sesgos significativos, puesto que no estipulan si los resultados se deben al tratamiento mediante electrolisis percutánea, o a las terapias que la acompañan.

6. Periodo de seguimiento. La extensión del periodo de seguimiento varía mucho de un estudio a otro ( 2 a 12 semanas). Un periodo de seguimiento mayor, consiente valorar la evolución de la patología, permitiéndonos saber si la recuperación continúa más allá del tratamiento aplicado, y la cuantía de tiempo que esta mejora en la sintomatología detectada. Esto ayudara tanto al diagnóstico como al tratamiento de las tendinopatías y poder, de esta manera, extrapolarlo a la clínica diaria. 


\section{Eficacia del electrólisis percutánea}

La revisión de los estudios encontrados, ofreció datos relevantes sobre la eficacia del electrolisis percutánea como terapia única, o combinado con otros programas terapéuticos, en el tratamiento tendinopatías del tendón de Aquiles y con patologías del tendón rotuliano.

Para el tratamiento de las tendinopatías rotulianas, la evidencia encontrada en esta revisión sistemática ofreció 28 artículos, de los cuales, 8 se emplearon en la revisión, concretamente, 5 estudios de series de casos y 2 estudios prospectivos en ratas y 1 caso clínico, los cuales solo tratan afecciones tendinosas de la extremidad inferior con electrolisis (4 de ellos trataban tendinopatías del tendón de Aquiles y los otros 4 se relacionaban con patologías del tendón rotuliano) (Tabla 1). Además, en ellos, el plan de tratamiento se basa en una terapia combinada de EPI con otros programas terapéuticos, lo cual limita la fiabilidad de los estudios.

\section{Propuestas para estudios futuros}

Los estudios examinados y valorados anteriormente, entregan unos resultados muy satisfactorios, aunque de una fiabilidad criticable. Teniendo en consideración las limitaciones encontradas en los artículos académicos e investigativos previamente nombradas, se hace ineludible la realización de investigaciones, en las que se estudie y estime la eficacia de la aplicación de electrolisis percutánea de manera exclusiva, y que utilicen un tamaño muestral mayor, con la ordenación de grupos de forma aleatorizada, y el establecimiento de un grupo en el cual se administre un placebo. Adicionalmente, sería recomendable, la ejecución de más estudios que constituyan de un periodo de seguimiento más amplio y detallado, permitiendo conocer el tiempo que se mantienen las mejoras, así como la recurrencia de la patología. Otro aspecto interesante a valorar, sería que cantidad de mejora se debe a la técnica de punción seca y cual, al electrolisis, por medio de un estudio que valore la eficacia de la punción seca (PS) frente al electrolisis percutánea (EP), o que dosis aplicada es más efectiva para cada tendinopatía tratada, por medio de estudios exploratorios y valorativos en el que se establezcan grupos con el tiempo se ha empleado e intensidades de aplicación diferentes.

Por otra parte, en los artículos valorados no se detecta la existencia de protocolos estandarizados para la aplicación del electrolisis, lo que manifiesta la gran variabilidad en el número de sesiones y tiempo de aplicación empleado (Tabla 1). De la misma manera, la existencia de protocolos estandarizados explicaría la variabilidad del tiempo de recuperación de la lesión, puesto que difiere significativamente de unos a otros trabajos. Por tanto, sugerimos la ejecución de nuevos estudios que consientan la elaboración de dichos protocolos y que proporcionen la práctica clínica.

\section{Conclusiones.}

- La presente revisión muestra que la electrolisis podría ser de significativa ayuda para acortar el número de sesiones necesarias para el tratamiento de las tendinopatías en la extremidad inferior y por tanto disminuir el tiempo de recuperación del paciente. A pesar 
de que la técnica no es indolora, la combinación de la misma con la realización de ejercicios excéntricos parece dar mejores resultados.

- Se necesitan más estudios que permitan establecer un protocolo de aplicación y una dosificación de la técnica estudiada, en cuanto a posición de los sujetos, número de sesiones, intensidad de la corriente, entre otros, que sea considerado válido, fiable y que se consiga aplicar en un tipo de tendinopatía especifica o en el tratamiento de este tipo de patología en general, por parte de un profesional especializado.

- Por último, hacen falta un mayor número de investigaciones y estudios con un alto grado de evidencia científica, con el fin de comparar la intervención con electrolisis percutánea con el efecto placebo, así como realizar más estudios en los que no se combinen varias terapias, con un seguimiento de los sujetos tanto a corto como a medio y largo plazo. Esto servirá para poder explorar y atribuir, única y exclusivamente al tratamiento con electrolisis percutánea, los resultados obtenidos en los sujetos tras la intervención, así como para poder evaluar el efecto que posee la técnica tanto a corto como a largo plazo.

\section{Referencias bibliográficas.}

Abat, F., Gelber, P. E., Polidori, F., Monllau, J. C., \& Sanchez-Ibaez, J. M. (2015). Clinical results after ultrasound-guided intratissue percutaneous electrolysis (EPI) and eccentric exercise in the treatment of patellar tendinopathy. Knee Surg Sports Traumatol Arthroscopy, 23(4), 1046-1052. doi:10.1007 / s00167-014-2855-2

Abat, F., Sánchez-Sánchez, J. L., Martín-Nogueras, A. M., Calvo-Arenillas, J. I., Yajeya, J., Méndez-Sánchez, R., . . . Gelber, P. E. (2016). Randomized controlled trial comparing the effectiveness of the ultrasound-guided galvanic electrolysis technique (USGET) versus conventional electro-physiotherapeutic treatment on patellar tendinopathy. J Exp Orthop., 3(1), 34. doi:10.1186/s40634-016-0070-4

Ackermann, P. W., \& Hart, D. A. (2016). General Overview and Summary of Concepts Regarding Tendon Disease Topics Addressed Related to Metabolic Disorders. Advances in Experimental Medicine and Biology, 920, 293-298. doi:10.1007/978-3-319-33943-6_28

Ackermann, P. W., \& Hart, D. A. (2016). General Overview and Summary of Concepts Regarding Tendon Disease Topics Addressed Related to Metabolic Disorders. Advances in Experimental Medicine and Biology, 920, 293-298. doi:10.1007/978-3-319-33943-6_28

Arias-Buria, J. L., Truyols-Domnguez, S., Valero-Alcaide, R., Salom-Moreno, J., Atn-Arratibel, M. A., \& Fernndez-De-Las-Pens, C. (2015). Ultrasound-guided percutaneous electrolysis and eccentric exercises for subacromial pain syndrome: A randomized clinical trial. Evidence-Based Complementary and Alternative Medicine, 01-09. doi:10.1155 / $2015 / 315219$

Arnal-Burró, J., López-Capapé, D., Igualada-Blázquez, C., Ortiz-Espada, A., \& Martín-García, A. (2015). Surgical treatment of chronic non-insertional Achilles tendinopathy in runners 
using bipolar radiofrequency. Revista Española de Cirugía Ortopédica y Traumatología, 60(2), 125-132. doi: 10.1016/j.recot.2015.10.003

Carvajal, F. Ó., Álvarez, P. D., Medina, M. F., \& Minaya, M. F. (2016). Efectos del electrolisis percutánea en el tendón rotuliano sobre la temperatura local y contralateral medida con termografía infrarroja. Revista Fisioterapia Invasiva, 1(1), 18-25.

Cook, J. L., \& Purdam, C. R. (2009). Is tendon pathology a continuum? A pathology model to explain the clinical presentation of load-induced tendinopathy. British Journal of Sports Medicine, 43(6), 409-416. doi:10.1136/bjsm.2008.051193

Cook, J. L., Rio, E., Purdam, C. R., Girdwood, M., Ortega-Cebrian, S., \& Docking, S. I. (2017). El continuum de la patología de tendón: concepto actual e implicaciones clínicas. Apunts Med Esport., 52(194), 61-69. doi:http://dx.doi.org/10.1016/j.apunts.2017.05.002

De Almeida, S. M., Da Silva, V. R., \& Ronzio, O. A. (2019). Surveillance on safety and complications four years after the introduction of Percutaneous Microelectrolisis (MEP®) Sport technique as a physical therapy practice. Fisioter Pesqui., 26(2), 213-218. doi:10.1590/1809-2950/18038726022019

De Carbo, W. T., \& Bullock, M. J. (2017). Midsubstance Tendinopathy, Surgical Management. Clinics in Podiatric Medicine and Surgery, 34(2), 175-193. doi: 10.1016/j.cpm.2016.10.006

De la Cruz, T. B., Albornoz, C. M., García, B. P., \& Naranjo, O. J. (2016). Autonomic Responses to Ultrasound-Guided Percutaneous Needle Electrolysis of the Patellar Tendon in Healthy Male Footballers. Revista Fisioter Invasiva, 34(4), 18-25. doi:10.1136 / acupmed-2015010993

De Souza, M. V., Osório, S. C., Da Silva, M. O., Martins da Costa, M. B., Dornas, R. F., Batista, B. A., \& Natali, A. J. (2015). Achilles tendon of wistar rats treated with laser therapy and eccentric exercise. Revista Brasileira de Medicina do Esporte, 21(5), 332-337. doi:http://dx.doi.org/10.1590/1517-869220152105144256

Freed, L., Ellis, M. B., Johnson, K., \& Haddon, T. B. (2019). Fasciotomy and Surgical Tenotomy for Chronic Achilles Insertional Tendinopathy: A Retrospective Study Using UltrasoundGuided Percutaneous Microresection. Journal of the American Podiatric Medical Association, 109(1), 01-08. doi:https://doi.org/10.7547/15-168

García, B. P., De La Cruz, T. B., Naranjo, O. J., \& Albornoz, C. M. (2018). Autonomic Responses to Ultrasound-Guided Percutaneous Needle Electrolysis: Effect of Needle Puncture or Electrical Current? Journal of Alternative and Complementary Medicine, ;24(1), 69-75. doi:10.1089 / acm.2016.0339 
Jennings, M. M., Liew, V., \& Marine, B. (2019). Updates in Tendinopathy Treatment Options. Clinics in Podiatric Medicine and Surgery, 34(4), 543-552. doi:10.1016 / j.cpm.2019.06.002

Korakakis, V., Whiteley, R., Tzavara, A., \& Malliaropoulos, N. (2018). The effectiveness of extracorporeal shockwave therapy in common lower limb conditions: a systematic review including quantification of patient-rated pain reduction. British Journal of Sports Medicine, 52(6), 387-407. doi:10.1136/bjsports-2016-097347

Lang, G., Pestka, J. M., Maier, D., Izadpanah, K., Südkamp, N., \& Ogon, P. (2017). Arthroscopic patellar release for treatment of chronic symptomatic patellar tendinopathy: long-term outcome and influential factors in an athletic population. BMC Musculoskelet Disord,18(1), 486. doi:10.1186 / s12891-017-1851-3

Lim, H. Y., \& Wong, S. H. (2018). Effects of isometric, eccentric, or heavy slow resistance exercises on pain and function in individuals with patellar tendinopathy: A systematic review. Physiotherapy Research International, 23(4), e1721. doi:10.1002/pri.1721

Lui, P. P. (2017). Tendinopathy in diabetes mellitus patients-Epidemiology, pathogenesis, and management. Scand J Med Sci Sports, 27(8), 776-787. doi:10.1111/sms.12824

Mani-Babu, S., Morrissey, D., Waugh, C., Screen, H., \& Barton, C. (2015). The effectiveness of extracorporeal shock wave therapy in lower limb tendinopathy: a systematic review. American Journal of Sports Medicine, 43(3), 752-761. doi:10.1177/0363546514531911

Mascaró, A., Cos, M. À., Morral, A., Roig, A., Purdam, C., \& Cooke, J. (2018). Load management in tendinopathy: Clinical progression for Achilles and patellar tendinopathy. Apunts Med Esport., 53(197), 19-27.

Mattiussi, G. 1., \& Moreno, C. (2016). Treatment of proximal hamstring tendinopathy-related sciatic nerve entrapment: presentation of an ultrasound-guided "Intratissue Percutaneous Electrolysis" application. Muscle, Ligaments and Tendons Journal, 6(2), 248-252. doi:10.11138/mltj/2016.6.2.248

Moreno, C., Mattiussi, G., \& Núñez, F. (2016). Therapeutic results after ultrasound-guided intratissue percutaneous electrolysis (EPI®) in the treatment of rectus abdominis-related groin pain in professional footballers: a pilot study. The Journal of Sports Medicine and Physical Fitness, 56(10), 1171-1178.

Ögüt, T., \& Yontar, N. S. (2017). Treatment of hindfoot and ankle pathologies with posterior arthroscopic techniques. EFORT Open Reviews, 2(5), 230-240. doi:10.1302/2058-5241.2 
Rodríguez, R. A., \& Mayordomo, A. R. (2017). Revisión sistemática de la eficacia de la electrolisis percutánea en el tratamiento de tendinopatías en la extremidad inferior. Revista Española de Podología, 28(2), 93-98. doi:https://doi.org/10.1016/j.repod.2017.05.002

Sánchez, A. J., Esquirol, C. J., \& Dalmau, S. I. (2016). La pregunta clínica y de investigación en Fisioterapia: el acrónimo PICO. Actualizaciones en Fisioterapia, XII, 80-83.

Sánchez, L. M., \& Seoane, P. R. (2018). Electrólisis percutánea ecoguiada y ejercicio terapéutico en tendinopatía de Aquiles: estudio de un caso. Fisioterapia, 40(6), 40(6), 331-225. doi:10.1016/j.ft.2018.07.005

Scott, A., Backman, L. J., \& Speed, C. (2015). Tendinopathy: Update on Pathophysiology. Journal of Orthopaedic \& Sports Physical Therapy, 45(11), 833-841. doi:10.2519/jospt.2015.5884

Shakked, R. J., \& Raikin, S. M. (2017). Insertional Tendinopathy of the Achilles: Debridement, Primary Repair, and When to Augment. Foot and Ankle Clinics, 22(4), 761-780. doi:10.1016/j.fcl.2017.07.005

Singh, A., Calafi, A., Diefenbach, C., Kreulen, C., \& Giza, E. (2017). Noninsertional Tendinopathy of the Achilles. Foot Ankle Clin, 22(4), 745-760. doi:10.1016 / j.fcl.2017.07.006

Splittgerber, L. E., \& Ihm, J. M. (2019). Significance of Asymptomatic Tendon Pathology in Athletes. Current Sports Medicine Reports (CSMR) | ACSM Journal, 18(6), 192-200. doi:10.1249/JSR.0000000000000600

Urrútia, G., \& Bonfill, X. (2010). Declaración PRISMA: una propuesta para mejorar la publicación de revisiones. Medicina Clínica (Barc)., 135(11), 507-511. doi: 10.1016/j.medcli.2010.01.015

Valera-Garrido, F., Minaya-Muñoz, F., \& Medina-Mirapeix, F. (2014). Ultrasound-guided percutaneous needle electrolysis in chronic lateral epicondylitis: short-term and long-term results. Acupuncture in Medicine, 32(6), 446-454. doi:10.1136/acupmed-2014-010619

Weber, J., \& Buchhorn, T. (2017). Midportion Achilles tendinopathy. Unfallchirurg, 120(12), 1038-1043. doi:10.1007/s00113-017-0411-5

Yang, Y., Lu, H., \& Qu, J. (2018). Tendon pathology in hypercholesterolaemia patients: Epidemiology, pathogenesis and management. Journal of Orthopaedic Translation, 6(16), 14-22. doi: 10.1016/j.jot.2018.07.003

Yeo, A., Kendall, N., \& Jayaraman, S. (2016). Ultrasound-guided dry needling with percutaneous paratenon decompression for chronic Achilles tendinopathy. Knee Surg Sports Traumatol Arthrosc, 24(7), 2112-2118. doi:10.1007/s00167-014-3458-7 
Zwiers, R., Wiegerinck, J. I., \& Van Dijk, C. N. (2016). Treatment of midportion Achilles tendinopathy: an evidence-based overview. Knee Surg Sports Traumatol Arthrosc., 4(7), 2103-2111. doi:10.1007/s00167-014-3407-5 
PARA CITAR EL ARTÍCULO INDEXADO.

Villamarín Arévalo, A. E., Muyulema Allaica, J. C., Culqui García, R. P., \& Sandoval Tulcán, M. E. (2019). La electrólisis percutánea y su eficacia en el tratamiento de tendinopatías en la extremidad inferior. Revisión sistemática. Ciencia Digital, 3(4), 210-226. https://doi.org/10.33262/cienciadigital.v3i4.966

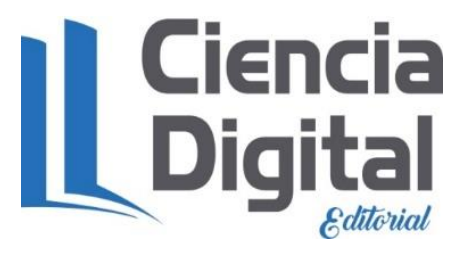

El artículo que se publica es de exclusiva responsabilidad de los autores y no necesariamente reflejan el pensamiento de la Revista Ciencia Digital.

El artículo queda en propiedad de la revista y, por tanto, su publicación parcial y/o total en otro medio tiene que ser autorizado por el director de la Revista Ciencia Digital.
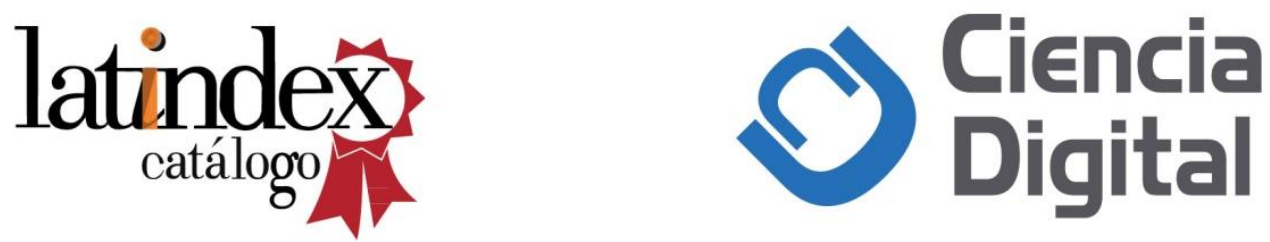